\title{
VIOLENCE EN ESPACES HYPER RÉELS
}

\author{
Dominique Crozat
}

\section{Armand Colin | Annales de géographie}

\author{
$2009 / 5$ - n 669 \\ pages 478 à 497
}

\section{ISSN 0003-4010}

Article disponible en ligne à l'adresse:

http://www.cairn.info/revue-annales-de-geographie-2009-5-page-478.htm

Pour citer cet article :

Crozat Dominique, « Violence en espaces hyper réels »,

Annales de géographie, 2009/5 n669, p. 478-497. DOI : 10.3917/ag.669.0478

Distribution électronique Cairn.info pour Armand Colin.

(c) Armand Colin. Tous droits réservés pour tous pays.

La reproduction ou représentation de cet article, notamment par photocopie, n'est autorisée que dans les limites des conditions générales d'utilisation du site ou, le cas échéant, des conditions générales de la licence souscrite par votre établissement. Toute autre reproduction ou représentation, en tout ou partie, sous quelque forme et de quelque manière que ce soit, est interdite sauf accord préalable et écrit de l'éditeur, en dehors des cas prévus par la législation en vigueur en France. II est précisé que son stockage dans une base de données est également interdit. 


\title{
Violence en espaces hyper réels ${ }^{1}$
}

\section{Violence in hyperreal spaces}

\author{
Dominique Crozat
}

FRE 3027 ART-Dev (ex-Mutations des Territoires en Europe), Université Paul Valéry, Montpellier

Résumé La peur. Peur du hun, du normand, du juif, du soviétique ou de l'arabe, toujours mythifiés, réinvention permanente du Barbare, mais aussi peur de toutes sortes de pestes reste un des moyens efficaces de consolidation du pouvoir de dirigeants peu scrupuleux. Notre époque se caractérise cependant par des changements majeurs : érigée en système de gouvernement, la propagation de la peur est pratiquée avec dextérité par les mêmes démocraties créées depuis deux siècles pour s'en affranchir. Surtout, la maîtrise des technologies de production de I'information et des médias de diffusion a remplacé avec efficacité la vieille rumeur artisanale. Construite par cette médiation qui la propulse dans I'hyper réel, cette peur place les populations sous la contrainte d'une violence permanente déstabilisante pour l'identité d'individus enfermés dans des territoires structurés par cette contrainte jusqu'à remettre en cause leurs fondements mêmes. Cet article se propose d'analyser les modalités de construction de ces espaces sous violence et les enjeux de cette vie en hyper réalité.

Abstract Living with fear, living on fear : historically, the fear of barbarians has always been exploited as a potent means of control (the mythical Hun, Norman, Jew, Soviet citizen or Arab...), as well as the fear of many different "plagues". Today, the democracies - once created to escape these means of constraint - use computer mediated communications and digitally virtual environments that have channelled these fears into realm of hyperreal information. This fear pushes people to accept and ask for permanent violence. The process produces a destabilization of the subjects' identity witch is in turn constrained into structured territories. In turn, this challenges their very foundations. This paper examines how these spaces are built under violence and life in hyperreality.

Mots-clés Espaces hyper réels, violence symbolique, identités spatialisées, contrôle social, politique de la peur, société du risque.

Key words Hyperreal spaces, symbolic violence, spatial identities, social control, politics of fear, risk society.

Processus de virtualisation du monde, des sociétés et de l'expérience des individus au moyen d'artefacts, performativité absolue de la narration, l'hyper réel est métaphore prise à la lettre, image qui ne fait plus sens mais devient le sens. Pour disposer d'un modèle opératoire, je distingue cet outil du virtuel, description banalisée et anodine reliée aux seules technologies. Développée à partir de Baudrillard (l'objectification du monde et la notion d'hyper réel) comme

1 Une version courte de ce texte est parue sous le titre Violência dos espaços hiper reais, in P. Joron (dir.), Comunicações da violência, Violências da Comunicação, Revista FAMECOS, $\mathrm{n}^{\circ} 29$, PUCRS, Rio Grande del Sul (Brésil), avril 2006, p. 45-54. http://revcom2.portcom.intercom.org.br/index.php/ famecos/article/viewFile/468/395. 
de Derrida (la performativité), la notion d'hyper réalisation recouvre l'ensemble des processus de création des espaces à partir de représentations qui coupent le lien direct qu'entretiennent l'espace réel et sa représentation. Omniprésents, qu'il s'agisse des espaces de la disneyfication (Eco, 1985) ou disneylandification (Brunel, 2006) du cinéma, des parcs à thèmes ou des re-constructions patrimoniales, du cyberspace (Kitchin, 1998), de la réalité virtuelle (McLellan, 1996), ces processus dessinent les contours d'un monde dont la mise en image prime sur sa réalité. À travers le passage du clochard au SDF, du terrain à la réalité reconstituée des SIG, de la campagne au rural, etc., l'hyper réel propose une réalité plus réelle, plus satisfaisante que le réel qui lui donnait origine ${ }^{2}$. Le recours à cette nouvelle espèce d'espaces hyper réels inclut les constructions réalisées au moyen des artefacts techniques producteurs de virtuel dans un ensemble croissant d'espaces plus immédiatement concrets car les uns et les autres ont en commun de prendre en compte en permanence leur médiatisation. Quand la culture devient médiaculture (Maigret, 2005 ; Macé, 2006), leur production se distingue par les remédiations qu'ils mettent en ouvre et leurs objectifs de construire un réel plus satisfaisant ; c'est ainsi le cas des nombreux espaces touristiques, par exemple les écomusées, ou de certaines fêtes locales destinées à créer un simulacre autonome de la ville. Les espaces hyper-réels modifient la perspective de l'interaction, interrogent le sens de l'insertion des sociétés dans l'espace et mettent en lumière un processus sociopolitique inquiétant analysé ici.

Cet imaginaire de la représentation, qui culmine et à la fois s'abîme dans le projet fou des cartographes d'une coextensivité idéale de la carte et du territoire, disparaît dans la simulation - dont l'opération est nucléaire et génétique, plus du tout spéculative et discursive. C'est toute la métaphysique qui s'en va. Plus de miroir de l'être et des apparences, du réel et son concept. Plus de co-extensivité imaginaire : c'est la miniaturisation génétique qui est la dimension de la simulation. Le réel est produit à partir de cellules miniaturisées, de matrices, de mémoires, de modèles de commandement et il peut être reproduit un nombre indéfini de fois à partir de là (Baudrillard, 1981, p. 10-11).

Ce processus de simulation et de multiplication d'artefacts autonomes à vocation commerciale génère une violence qui s'exerce à trois niveaux. Tout d'abord la diffusion par les médias de scènes brutales : selon une enquête de 1995, les six chaînes de télévision française diffusaient en moyenne une mort violente toutes les 12 minutes. À un second niveau, le virtuel est prétexte d'une régression sociopolitique (Miller, 1997). Les mondes abstraits des modèles économiques ignorent leurs implications sociales réelles : interventions du FMI, délocalisation de services en ligne vers des pays à main-d'œuvre bon marché, régression des services publics en zone rurale sous prétexte de connexions internet plus faciles,

2 Pour une définition voir Crozat (2007a); une synthèse plus complète sur la question est en cours d'achèvement (La production des espaces hyper réels. L'utopie d'un monde parfait, parution 2010). 
utilisation des technologies de l'information pour renforcer le contrôle des populations...

Ces deux premiers niveaux ne sont abordés ici qu'à travers leurs liens avec la troisième violence, consubstantielle au processus d'hyper réalisation : sa logique profonde pousse à la disparition de la dimension symbolique du monde (Baudrillard, 1968 ; Stiegler, 2004) induit un sentiment de dé-territorialisation, de perte des repères spatiaux socialisés, une confusion identitaire douloureuse. Pour Umberto Eco (1987), l'ordre imaginaire de Disneyland est opposé au reste du monde, réputé réel, mais, en fait, l'Amérique toute entière et le monde, sont la simulation hyper réelle. "Ce n'est plus une question de fausse représentation de la réalité, mais de dissimulation du fait que le réel n'est plus réel... » (Baudrillard, 1981). Ce «crime parfait» (Baudrillard, 1995) n'est pas abstrait: en 2004, deux enfants anglais sont morts déchiquetés par les ours d'un zoo après s'être introduits dans leur cage; nourris de dessins animés, ils connaissaient seulement des nounours sympathiques.

Le mal-être qui en découle accompagne l'hégémonie de la transparence, conditionne l'appréhension sociale des espaces et détermine leur aménagement : de l'installation de caméras de surveillance dans les espaces publics à la construction de risk-free environments (Spring, 2004), mais aussi la privatisation de l'espace public (Razac, 1999 ; Rodriguez, 2005), l'essentiel de l'action des gouvernants déstabilise les relations sociales et le fonctionnement des démocraties (Davis, 1997, 2006).

Cet article pose deux questions : en quoi la production du social localisé estelle affectée par la généralisation de ces espaces métaphorisés violents ? Comment réinstaller le politique évacué du processus majeur de production de l'espace des sociétés ? Après avoir considéré comment la manipulation de l'événement offre à chacun une expérience médiatisée du monde, nous verrons ensuite en quoi, du tsunami au changement climatique, du terrorisme à la délinquance ou toutes sortes de peurs plus immédiatement personnelles construites par l'instrumentalisation du risque, cette violence amène à intérioriser la peur comme structurant majeur de nos sociétés (Beck, 1996) : dans ce schéma, le rêve de la sécurisation totale du monde passe par sa mise à distance pour construire, au final, un monde réactionnaire niant l'individu et la représentation politique.

\section{L'expérience médiatisée du monde remplace l'interaction}

«L'hyper réel est le concept d'une géographie simulée » (Rodaway, 1994) : ces simulations ne sont pas des représentations, copies imparfaites d'un original ; plus claires, complètes, elles sont meilleures que l'original : "c'est désormais la carte qui précède le territoire - précession des simulacres -, c'est elle qui engendre le territoire » (Baudrillard, 1981, p. 10). Ce fut toujours le cas, mais cette représentation était couplée à une expérience concrète du terrain; dans l’hyper réel contemporain, cette expérience est elle-même médiatisée. 
La technologie devient «[...] magique. Ainsi $[\ldots]$ les techniques des Systèmes d'Information Géographique (SIG) personnalisent une abstraction, une vue scientifique du monde, si bien qu'on a l'impression qu'elle est engagée dans le processus de génération des relations spatiales» (Sack, 1997, p. 276-22). Alibis d'une gouvernance sans lien avec la réalité citoyenne, ils transforment la décision politique en processus technique et disqualifient par avance toute initiative démocratique en créant un fatum qui rend impossible une action volontaire.

Une partie de la planète : l'Afrique (Shields, 2003, p. 81), les espaces de loisirs (Rodaway, 1994), les périphéries urbaines (Crozat, Raibaud, 2009), etc., n'est perçue qu'à travers des reconstructions hyper réelles. Les producteurs de virtuel social (sondages d'opinion, presse) recherchent des individus représentatifs mais, en fait, hors de la norme (pompier héroïque, rescapé providentiel, leaders...). Aubenas et Benasayag (1999) montrent que l'information destinée au grand public est structurée autour du récit de catastrophes ou massacres, s'efforce d'en dresser des bilans chiffrés. Au nom de la transparence, elle ignore les phénomènes complexes ou le quotidien banal. Cette production du monde devient hyper réelle quand elle modifie la vie des populations : l'information destinée au grand public sur des pays comme le Botswana ne provient quasiment que d'émissions télévisées idéologiquement connotées (écologie, développement durable) telle Okavango de Nicolas Hulot (Perrot, 1997). Rossi (2001, p. 59 et suiv.) montre qu'en Namibie, les populations aborigènes doivent modifier radicalement leurs modes de vie traditionnels. Le gouvernement l'exige, poussé par les voyagistes qui vendent des séjours écologiquement corrects. En Ouganda, l'Union Européenne finance puis félicite le gouvernement pour avoir agrandi le territoire de pâture des éléphants au prix du déplacement de 30000 personnes.

Le changement climatique, la forêt amazonienne ou le tsunami indonésien de 2005 subissent cette reconfiguration : dans ce dernier cas, en quelques jours, les médias ont créé un produit, l'ont nommé ${ }^{3}$ et l'ont vendu : on imagine l'ampleur des gains publicitaires suscités par ces émissions/émotion. Ils ont aussi utilisé les ONG, pour donner à vivre l'expérience du monde au plus grand nombre (vibrons ensemble) en insistant sur la prétendue visibilité de l'argent donné : comme pour un steak ou pour l'argent déposé sur un plan épargne retraite, chacun doit connaître sa traçabilité ou régime de mise en visibilité virtuelle.

La tentative de recréer l'événement à partir de la grippe aviaire n'a pas eu le même succès, probablement parce que la manipulation était trop poussée : en voulant créer une psychose à partir d'une maladie qui n'existe pas encore et n'existera peut-être jamais, les arguments frôlent parfois le ridicule quand The Independant de Londres (15 novembre 2005) titre en première page : " the world's only weapon against bird flu ${ }^{4}$ sous une photo montrant un graine de badiane ou anis étoilé ; en 2003, la société Pernod-Ricard avait utilisé presque

3 Auparavant, hormis les géographes, qui connaissait les tsunamis?

4 La seule arme mondiale contre la grippe aviaire. 


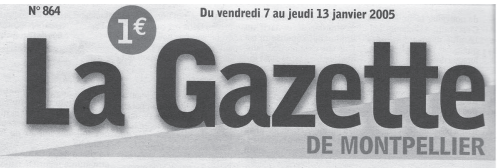

-Les conséquences seraient moins désastreuses
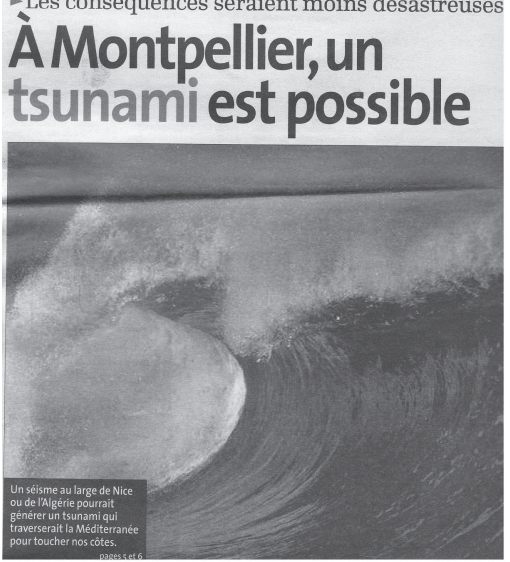

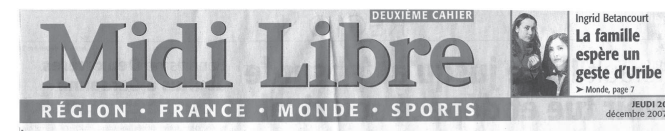

Risque naturel La Méditerranée sous la menace des tsunamis

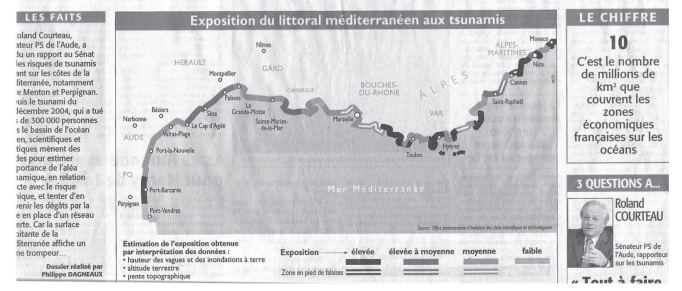

Fig. 1 : Le ridicule ne tue pas, il rend docile par la peur qu'il suscite (Jameson, 2007b). Le tsunami de Montpellier ou la fabrication de l'hyper réel avec une photo du Pacifique (La Gazette): un mois plus tard, une simulation des réactions des services de sécurité à ce type d'événement fut entreprise. Un récent rapport parlementaire (Midi Libre) tente le même discours sécuritaire : finalement, excepté en quelques points limités (essentiellement de Nice à Antibes), sur le littoral méditerranéen français, les vagues ne devraient pas dépasser 60 centimètres (risque moyen) à un mètre (risque élevé), avec une occurrence potentielle séculaire...

La Gazette de Montpellier, 7/01/2005 ; Midi Libre, 20/01/2007

la même photo pour la publicité de printemps de son pastis fabriqué avec l'anis étoilé...5

L'ensemble des rapports à la nature est au cœur de ces manipulations (fig. 1). Dès l'abord, le passage de la notion de milieu à celle d'environnement est signe d'une mutation complète (Virilio, 1984), l'entrée dans l'hyper réel : on " réalise » l'environnement comme un programme d'immeubles et on transforme la nature en un registre de signes.

Un registre, contrairement à un code, ne s'articule pas sur le principe d'opposition, il se contente d'empiler les données pour les soumettre à un programme de traitement (appelé logiciel au niveau de l'utilisateur). Plusieurs registres (scientifique, politique, publicitaire, touristique, agricole, médical...) sont ouverts, permettant des connexions transversales. [...] Nous ne sommes plus dans un rapport symbolique de destruction/protection, celui défini par la relation avec la nature, nous sommes dans un rapport

5 Article écrit en 2006 et 2007. Après trois ans d'attente, la grippe A est enfin arrivée. Cette pandémie médiatique fait l'objet d'un déploiement considérable de moyens, y compris dans des pays pauvres qui éprouvent des difficultés pour en mobiliser autant contre des maladies autrement plus répandues et mortifères (diarrhées infantiles ou paludisme). En Europe des ailes entières d'hôpitaux ont été réquisitionnées (Montpellier, Hambourg) alors qu'on peine a assurer les soins de base pour l'ensemble de la société. Comme le bug de l'an 2000, cette pandémie médiatique est un prototype du « complot sécuritaire » de Jameson (2007b, p. 102-103) qui permet d'occulter des problèmes réels en s'assurant une totalisation de l'attention. Sur l'ensemble de la planète, en quatre mois d'existence, elle a fait moitié moins de victimes que les accidents de la route en France pendant la même période, autant qu'une semaine de suicides en France, 0,03 \% de la surmortalité due au manque de réactivité des systèmes de santé de l'Europe de l'Ouest face à la canicule de 2003. Mais ce réel-là est si dérisoire... 
de communication (interactive) avec l'environnement. Il n'est plus guère possible de toute façon de distinguer le contexte signalétique du contexte dit «naturel ». La forêt, par exemple, se voit affublée de pancartes ou de flèches qui balisent l'aventure signalétique que représente désormais une promenade dans les sous-bois avec une carte d'état-major en permanence sous les yeux (Gautier, 2002, p. 65).

Révélatrice est l'évolution de la notion de "sauvage »; domaine de l'anti-social par excellence, le sauvage énonce le partage des espaces selon une dichotomie violence/pacification : tiré du latin selva, la forêt, le sauvage s'oppose à domus, la maison et le domestique. À ce titre, c'est ce qui menace la culture (Micoud, 2001): le terme caractérise l'animal ou le milieu qui n'est pas maîtrisé par l'homme et peut lui nuire. Or, aujourd'hui, le «sauvage polysémique » (Bobbé, 2001) décline trois types nettement caractérisés.

Le premier initie le passage de l'espèce à la population. Usuellement, le mot espèce est relié à un milieu et ne réfère pas à un espace précis. L'évolution récente marque la territorialisation d'un groupe car le terme réfère au dernier animal tué dans un territoire (loup ou ours) qu'on fait revivre, met en scène pour construire son mythe : le continuum historique permet la réintroduction ; c'est le sauvage under control. Mais l'animal réintroduit n'est pas vraiment sauvage puisqu'il est localisé en permanence, vacciné, nourri afin de le sédentariser sur un territoire et limiter sa prédation. Ce sauvage anthropisé a pour fonction de donner une labellisation naturelle à un territoire.

Le second type du sauvage contemporain correspond au passage de nuisible à " momentanément (et localement) proliférant » (Bobbé, 2001) ; l'écologisation vise un équilibre entre le sauvage et son milieu. C'est la fabrication maitrisée d'un sauvage spontané (les loups dans les Alpes françaises). Cette gestion naturalisante implique la territorialisation, localement et en relais avec un réseau européen de protection de l'espace (Natura 2000, Directives Oiseaux, Directive Habitat...). L'apparente plus grande liberté de l'animal masque une surveillance stricte (élimination sélective des éléphants dans le Parc Kruger en Afrique du Sud).

Dans le troisième type, Bobbé passe au sauvage ordinaire, le véritable sauvage : les chiens errants. L'importance croissante du phénomène ${ }^{6}$ n'empêche pas de l'ignorer, car il est perturbant puisqu'il pose l'échec de la domestication. On parle donc de régulation; l'animal sauvage est bon à gérer mais n'est pas médiatisé et donc inexistant.

Ainsi la reconstruction hyper réelle de l'expérience vécue en évacue le symbolique. Nous allons voir que, dans toutes ses dimensions, de l'intimité du corps jusqu'à la définition de l'urbanité, cela génère une désappropriation douloureuse de leurs territoires par les populations.

615000 chiens abandonnés chaque année en France ; 10 fois plus divaguent en permanence ; un demi million de cas d'agressions sont traités par les assurances, la gendarmerie ou la police ; en moyenne, la Poste recense plus de 3200 arrêts de travail pour morsure... 


\section{La mort du symbolique}

Plus que le réel, la question centrale concerne l'expérience : comment donner existence intime au monde pratiqué ? De l'échographie (Fournand, 2007) à la généralisation du GPS, dans l'Art, la géo-graphie des espaces hyper réels est expérience du monde pour des sujets devenus consommateurs, expérience sensuelle (Rodaway, 1994) in-corporée (Crang et alii, 1999) ou incarnée (De Barros, 2003) :

Dans le Body Art et la Performance des années 1960 et 1970, le corps était l'instrument d'une revendication sociale ou politique, d'une émancipation soufferte, le lieu physique et conceptuel d'une exploration inquiète des limites (la douleur, la nudité, le gender, l'opposition public/privé...). Aujourd'hui, le contexte a changé et le corps de l'art, loin d'être une surface inerte et indifférente, se définit comme une membrane, qui filtre nos mutations. Comme une interface mobile qui reflète les relations (adaptatives, osmotiques, conflictuelles...) avec l'environnement. Au fond ce qui a changé, ce n'est pas le corps, mais l'environnement. Médiatisé, technologisé. De la société du spectacle à l'ère de l'information. La mutation du contexte implique un déplacement des frontières : les médias, les technologies ne se limitent plus à capter, à représenter le corps, mais commencent à l'investir, à le modifier, à se mixer aux tissus organiques, à devenir vecteurs de subjectivation et d'hybridation. Nous avons oublié Foucauld pour passer à Baudrillard. (Quinz, 2003, p. 187)

Privé de représentation de l'unité du sujet par la génétique, le clonage, la chirurgie esthétique, les dispositifs numériques de virtualisation ou les réseaux, le corps n'est plus aussi central. Il devient entre-deux identitaire complexe, structure ouverte et modifiable, champ d'interaction entre une pluralité de dynamiques. La reproduction identique du même, à volonté et ad libitum fait passer de "qu'est-ce que le réel ? " à «suis-je réel ? ». Le dépôt d'une image de soi sur les réseaux empêche d'en prévoir les usages ou détournements possibles : «toute image saisie par le réseau peut être indéfiniment reprise, manipulée, falsifiée, remise en forme à notre insu» (Quéaud, 1993, p. 46). L'artiste Eduardo Kac exhibe Alba, son lapin transgénique fluorescent, et propose de créer à la demande des animaux de compagnie uniques (De Barros, 2003). Il a créé aussi Le huitième jour, un microcosme artificiel : sous un dôme de verre, des souris, des poissons et des plantes transgéniques initient le microcosme paradigmatique d'un nouveau monde, alimenté par un robot manipulé par Internet. Les jeux vidéo (Sims) ou la télé réalité ont la même ambition. "Le sujet s'appareille désormais à des automatismes d'une puissance inouïe " (Couchot, 1998, p. 12).

Déstabilisé, l'homme est acculturé progressivement à l'utopie d'une création sans aléatoire; dans l'émission Okavango, Nicolas Hulot s'arroge le droit de trancher entre la culture et la nature (Perrot, 1997). Il construit un monde autour d'un détail pertinent (Jameson, 2007b, p. 60), c'est-à-dire ses qualités 
filmogéniques : les populations importent moins que la mise en image. Incapables de distinguer le réel de ces simulations, les spectateurs les assimilent comme une expérience. Cette «schizophrénie où l'expérience vécue serait indiscernable de l'expérience virtuelle, où toutes les frontières entre le réel et l'imaginaire seraient caduques » (Jolivat, 1995, p. 114) débouche sur «Les Antilles à Jonzac » (Charente-Maritime), la campagne sur l'aire d'autoroute nommée Ruralie, près de Niort. L'expérience du monde est médiatisée selon la logique des promoteurs de centres commerciaux : pourquoi aller ailleurs puisque nous vous vendons ici une réalité plus complète ? Ainsi l'hyper réel ne quitte pas le réel mais il l'assimile. « La simulation n'est pas une procédure innocente [...]. Elle reconfigure l'ensemble des domaines sociaux selon ses propres modalités de confusion, d'indistinction qui empêchent l'éclat de la riposte, qui troublent singulièrement l'intelligibilité, qui orientent vers un consensus mou » (Gauthier, 2002, p. 233-234). Le projet de rendre visible « l'au-delà des apparences sensibles " (Mandelbrot) s'étend à la subjectivité. La logique profonde de l'œuvre d'Alan Tuning est la création d'un ordre utopique absolu (Breton, 1992) : le monde du surhomme que chacun mérite d'être. Cette généralisation du calcul au-delà de la sphère productive, submerge l'ensemble des dispositifs d'individuation psychique et collectif. Ce " nouveau stade, quoique hors du commun, de la perte d'individuation, en relation avec un nouveau stade de l'histoire des machines et donc de la technoscience, est aussi le nouveau stade d'un processus de grammatisation» (Stiegler, 2004, p. 100).

La pire violence de l'hyper réel réside dans cette dépossession, misère symbolique déstabilisante. Anne Fournand (2007) montre ainsi à travers la construction d'une spatialité corporelle au moyen de l'échographie foetale comment l'introduction de l'image a permis une mise à distance de la mère par rapport à son futur enfant. La promotion forcenée de cette transparence participe ainsi de l'élimination d'une part d'ombre majeure dans nos sociétés : nous sommes dans une écologie du sujet appareillée, d'un sujet dont les limites entre le corps et le Soi sont confuses (Pile et Thrift, 1995) : certains établissements scolaires développent le fichage-repérage des élèves au moyen de leur empreinte de main. Une école est un lieu de construction de l'identité, d'interconnaissance maximale et de re-connaissance; l'utilisation de telles technologies peut apparaitre comme un viol de l'intimité profonde mais remet aussi en cause le projet collectif sur lequel se fonde l'institution. La même question se pose au sujet des personnes dépendantes (Percival, Hanson, 2006).

La relation à l'habitat et aux lieux évolue sous l'influence des mutations de notre société (mobilité, flexibilité) et des innovations techniques (Jameson, 2007a ; Olalquiaga, 1992). L'hyperspécialisation des fonctions spatiales induit un appauvrissement relationnel (Lopez Levi, 1999). La standardisation technique, esthétique et économique des paysages et le désir compulsif de sécurité provoquent une dépendance à l'égard de l'expertise technique et de l'aménagement qui déresponsabilise leurs habitants (Sennett, 1991) car réducteurs de l'aspect affectif et éthique des lieux. La «MacDonaldisation du monde » ou asservissement du désir à des logiques consuméristes (Châtelet, 1997 ; Stiegler, 2004) et 
la transparence permanente produisent des suburbanites, "armées d'hommes et de femmes à l'organisation clonesque» (Relph, 1981).

Jadis, culture localisée dans le temps et dans l'espace, le lieu devient référent générique. À l'instar des espaces centrifuges des villes américaines (Baudrillard, 1988), assemblage d'éléments normés tirés de différents espace-temps et infiniment dupliqués, la multiplication des GPS (automobile, téléphones portables) permet de traverser ces non-lieux (Augé, 1992), sans avoir à les re-connaître. Culture, urbanisme, marketing, administration des territoires et politiques publiques se confondent dans la production d'événements selon une expertise technique uniforme qui les substitue aux systèmes symboliques (Crozat, 2004 ; Fournier et alii, 2009). Cessant d'être un agent informé mais « une collection de caractéristiques, quelque chose qui répond à des stimuli spécifiques en rapport à des comportements attendus » (Rodaway, 1995), le consommateur/(télé)-spectateur remplace l'habitant/citoyen.

La déréalisation du monde par déshumanisation du processus de création des lieux génère un malaise, la psychesthénie (Olaquiaga, 1992), "désorientation » (Jameson, 2007a) ou condition post-moderne (Soja, 2000) : perturbé dans sa relation personnelle avec le territoire environnant, le sujet est incapable de localiser ses frontières corporelles. Il confond donc ces paramètres physiques et les espaces représentés ; il joue d'identités spatiales différenciées, fuit dans l'imaginaire sans échapper à cette illusion de l'interaction au monde car les modèles proposés (centres commerciaux, parcs d'attraction, télévision, etc.) sont valorisés comme unique réalité.

Mais Olalquiaga note l'émergence d'espaces de subversion : des groupes travestissent les lieux avec les mêmes outils. "Radicalisme ironique » et retour du politique, la réinvention de la culture latino à Los Angeles (Price, 2000), Superbarrio $^{7}$ à Mexico ou le sous-commandant Marcos, le mouvement punk festif chilien; en Espagne Muchachito Bombo Infierno, en France le groupe Mano Negra et ses avatars s'inscrivent dans cette logique, comme le cyberworld : jeux vidéos, cyberpunk (Bell, 2001), art électronique. Si la révolte devient rare (Châtelet, 1998), pour la plupart des individus, ce désir fondé sur l'angoisse génère tristesse (Lipovetsky, 2004), ivresse et effroi (Missonnier, Lisandre, 2003) et une demande de sécurisation, fondement d'un monde réactionnaire. Jouer avec Big Brother (McGrath, 2004) n'est pas si facile.

7 Personnage fictif en tenue de catcheur, entre Superman et Super Mario, né après le séisme de 1985. Anti-héros et Robin des Bois, c'est un père de famille des quartiers d'habitat précaire qui découvre I'action sociale (lutte contre le SIDA) ou politique : membre de l'assemblée des quartiers en lutte contre les politiques hyper libérales, il bloque des banques ou la bourse (Schwarz, 1994). En 1987, il est I'emblème de campagne de Cuauhtemoc Cardenas, candidat des pauvres. En 1996, aux USA, suscitant les mêmes réactions que Coluche en 1981, il devint pseudo-candidat des wet-backs (immigrants clandestins) aux élections présidentielles. 


\section{L'utopie d'une sécurisation du monde par sa mise à distance}

Le monde hyper réel est sécurisé selon deux logiques. Tout d'abord, une contextualisation du risque dans les systèmes socio-spatiaux; le risk-free environment (Spring, 2004) ou la Privatopia 8 y répondent. Par ailleurs, comme toute utopie, ce monde d'ordre vise la perfection du dessin et la totalisation, stérilise l'imaginaire au nom de la transparence (Davis, 1997). Ainsi en est-il du petit monde ordonné de l'univers des jeux vidéo (Schmoll, 2000, p. 43-44 ; Shields, 2003, p. 130), représentatif d'une société où «[...] force est en effet de constater que l'idée de qualité et de bien-être, suivant l'évolution des sociétés occidentales et des valeurs que celles-ci véhiculent, a évacué tout ce qui n'est pas prévu, pas contrôlable, tout fatalisme en quelque sorte. [...] un refus de l'incontrôlable comme de l'inattendu. [...] le risque - vocable dont la charge sémantique est tout de même forte - a élargi sa portée et concerne des pans entiers des rapports homme-nature et homme-homme qui n'étaient pas, jusqu'alors, perçus comme risqués » (Reynaud, 2006).

Cette obsession est à l'origine de l'utopie totalitaire des « archipels carcéraux » métropolitains (Soja, 2000) organisée autour de l'idéologie de la transparence et du «retour du camp » (Minca, 2005) : il existe déjà 260000 caméras de surveillance à Pékin, 400000 d'ici les Jeux Olympiques de 2008. Interrogée par la télévision (Philippe Rochot pour la chaîne A2), une habitante trouve cela normal pour préserver «la stabilité de la société ». Cette banalisation explique sa généralisation ; en 2005 à Montpellier, le budget municipal "Gestion citoyenne " recouvre l'aménagement de la Maison de la démocratie $(0,3 \mathrm{M} €)$ et surtout «l'extension du réseau de surveillance vidéo urbaine » (1,2M€, soient 1,2\% de l'investissement total ou $5 €$ par habitant). Dans les lieux publics du RoyaumeUni, on passe de 1 à 25 millions de caméras entre 1997 et 2007 ; un londonien est filmé 300 fois par jour, sans contrôle de l'usage des données recueillies. Pour lire autant de caméras, l'INRIA, à Grenoble, travaille sur des logiciels d'analyse automatique de comportement. Mais les caméras n'ont pas empêché les attentats terroristes londoniens de 2006 ni fait baisser la criminalité sur la voie publique.

8 L'émergence de formes de gouvernements privés (Saunier, 2004) des gated communities et cerradas en sécession urbaine (Le Goix, 2001 ; Capron, 2006) construit la privatopia de McKenzie (1994). C'est l'ambition d'une définition complète du monde face aux incertitudes du réel car « la réalité a perdu son référent, et les modèles, simulations et discours tendent à devenir plus que réels et se confondre à la réalité elle-même, c'est-à-dire, une hyper réalité caractéristique du développement du capitalisme tardif » (Rodriguez, 2005, p. 130). Cette quête de l'entre-soi (Chevalier, Carballo, 2004) fonctionne selon une logique de club (Rifkin, 2000) : un groupe coopté avec des niveaux sociaux et des modes de vie proches, fermé par refus de la diversité du monde " puisque l'accès réservé aux membres partitionne la population entre bénéficiaires et non bénéficiaires » (Perrin, 2007). Aussi, la privatopia construit un espace sécurisé, une architecture qui «matérialise la peur » (Rodriguez, 2005, p. 127). Cette « utopie qui exprime l'échec de l'utopie »(Bernal, 2005, p. 166) institue une disciplinarisation de l'espace urbain par sa transparence totale. 
« On renverse la charge de la preuve en considérant que chaque citoyen est un suspect potentiel $»^{9}$. Sans débat démocratique, la généralisation de ces processus de production disciplinaire de l'espace remodèle la ville (Klauser, 2004) : la société industrielle est transformée en société $d u$ risque. Ce concept désigne une phase du développement de la société dans laquelle «la dynamique du changement de production des risques politiques, écologiques et individuels échappe de plus en plus aux institutions de contrôle et protection de ladite société industrielle. » (Beck, 1996, p. 201). Cette «écologie de la peur» (Davis, 2006) est mise en valeur par la crise des banlieues françaises de 2005 : en perte de vitesse, un ministre proche de l'extrême-droite provoque le déclenchement d'émeutes amplifiées par leur médiatisation. Lorsqu'elles décroissent, il les instrumentalise par l'institution de l'état d'urgence. Logiquement (Jameson, 2007b), il gagne puisque, dans les sondages, sa côte de popularité remonte rapidement, mais l'état d'urgence, par définition non démocratique, reste en vigueur pendant deux mois et n'empêche pas de nouvelles émeutes dans la nuit du 31 décembre 2005... Les modes de régulation sociale et culturelle (la sécurisation et la consommation) imposent un ordre nouveau, ce modèle du capitalisme culturel de Rifkin (2000) où le marketing contrôle les consciences et les corps par la machinisation de la vie quotidienne : pour Rodaway (1994, chap. 9) la mise en spectacle généralisée produit les normes que le télé-citoyen est prié d'assimiler en permanence sous peine d'être marginalisé, propulsé hors de la «bonne » société, société de l'accès de Rifkin. L'idéologie de la communication produit cette "paix sociale cybercivile " (Châtelet, 1998). L'univers des représentations médiatiques a absorbé l'ensemble des activités humaines, tout propos à ce sujet en vient et est donc biaisé ; le débat démocratique n'a plus pour fonction que d'accompagner les modalités de mise en pratique de ces processus de contrôle jouant sur le binôme peur/désir de consommer.

L'idéal utopique impose ainsi une société totalement lisible. Ce fond de commerce de tous les totalitarismes (Foucault, 1975) induit codages et fichage, fluidité et lisibilité, cryptage et enfermement, mise à l'écart, homogénéisation et mercantilisme du désir ; l'homme nouveau n'a pas de secret. Le centre commercial (Levi, 1999), les systèmes de vidéos surveillance des gated communities et cerradas (Razac, 1999) jusqu'aux repas dansants du périurbain français (Crozat, 2004) ambitionnent une définition complète du monde face aux incertitudes du réel : la privatopia prend le dessus sur la cité publique; nous assistons à « une transformation sinistre et homogène de l'espace. Un nouveau type d'urbanisme manipulateur, dispersé et hostile à l'espace public traditionnel, est en train d'émerger dans les centres et périphéries de nos villes. Ce sont des espaces dans lesquels tout est organisé pour obtenir un contrôle absolu et où l'idée d'interaction authentique entre les citadins a disparu » (Rodriguez, 2005, p. 128).

9 Jean-Pierre Manach, de Big Brother Awards (branche française de Privacy International), Le Monde, 5 août 2005 
La matrice reste sous contrôle (Imken, 1999) au profit de pouvoirs qui en instrumentalisent l'évolution.

\section{Construire un monde réactionnaire}

La lisibilité totale et le contrôle seraient l'antidote sécurisant face à l'individualisation de la société (Giddens, 1994; Beck, 2001) et la recomposition d'un symbolique à basse densité (Sennett, 1991; Olalquiaga, 1992). La peur de l'inconnu - insécure par définition — induit alors le conservatisme. L'imagination créatrice est impossible puisque l'hyper réel ne reproduit que ce qui est déjà représenté (Gauthier, 2002) : le conformisme de Lara Croft ou Matrix n'inaugure pas une autre subjectivité (Couchot, 2003). C'est la contradiction majeure : loin de la métaphysique, l'idéologie commerçante et technologique hyper réelle n'a qu'une ambition stérilisante de boutiquier (Eco, 1985) qu'on peut qualifier de réactionnaire car elle fonctionne sur deux niveaux : politique et moral.

Politiquement, gardiennage, surveillance, filtrage systématique et cloisonnement des sociétés (Davis, 1997) structurent ce monde idéal du refus du conflit social et de l'invention de nouvelles ségrégations par la négation de l'Autre, mais pas n'importe quel Autre : le monde hyper réel est cloisonné pour une "élite" qui nie ses pauvres, condamne les sans-logis à l'hétérotopie (Don Mitchell, 2001, p. 64) ; de leur côté, les «sous-privilégiés » de Hong-Kong, "économiquement faibles » d'Europe sont confinés dans des circuits économiques et de loisirs spécifiques (logement social, bars populaires, supermarchés Lidl ou Aldi, etc.) à l'écart du reste de la ville quand les plus riches accèdent au commerce en ligne (Metton, 1998). De même, l'image sociale construite dans les écomusées nie le conflit et esthétise la misère (Cantau, 2003). Ainsi, l'âge des loisirs n'est pas une libération ; il permet surtout de renforcer le contrôle social (Corbin, 1995).

Ce monde ondulatoire incorpore les mouvements d'opinion ou de fascination et est régi par un dispositif idéologique (Williams, 1996, p. 67), un méta-récit à travers lequel s'exprime la réalité froide et nue du pouvoir social (Foucault, 1975, p. 196). Parler de postmodernité n'a donc pas de sens : les anciennes structurations du pouvoir ne sont pas remises en cause ; seule évolue leur mise en ouvre. Vendus à l'origine pour sécuriser les établissements scolaires contre les intrusions, les systèmes de fichage des empreintes de mains des élèves ont été détournés de leur usage. Ils sont utilisés soit pour connaître l'absentéisme en temps réel, soit, installés dans les réfectoires, pour dissuader les élèves qui auparavant mangeaient en utilisant la carte de cantine d'un de leurs amis : le prétexte sécuritaire cache une finalité sociale discutable.

La «guerre» aux pauvres (Don Mitchell, 2001, p. 57) reste donc la norme mais les modalités de conflit social ou politique évoluent : comment à ce premier niveau réintroduire la cité, apprendre la manipulation de la société hyper réelle ? Il ne saurait suffire de promouvoir « une ré-existence, autre manière d'exister pour et dans la cité, dans laquelle les perspectives citoyennes seraient 
en accord avec les désirs et plus seulement avec les « dessins » planifiés par les secteurs/segments les plus élevés de la gestion urbaine » (Lima, 2005, p. 85).

En effet, à un second niveau, l'hyper réel est un projet de société moralement réactionnaire qui nie l'individu moderne autonome et responsable : l'omniprésence des réseaux cybernétiques questionne ainsi la responsabilité de la manipulation des images déposées et de ceux qui agissent ces réseaux. Les virus sont aussi une forme de vandalisme que le virtuel encourage, par son abstraction et l'apparente immunité de l'anonymat. Plus souvent « inspiré » que révolutionnaire, le discours des créateurs de virus traduit le retour d'un divin de pacotille :

Le réel peut se transformer à nos yeux enfin dessillés, si nous savons déceler en lui la main d'un maître programmeur, d'un hacker divin, dotant ses créatures de la liberté d'évoluer et de se transformer en fonction de leur approfondissement et de leur compréhension du "programme ». [...] derrière l'apparence des choses il y quelque chose d'insoupçonné, d'incroyable, de merveilleux, qui se tient tapi, caché, incommensurable. Derrière le monde, ou au-delà de lui, il y a un autre monde (Quéaud, 1993, p. 47).

Sloterdijk (1996) y ajoute la disparition de l'homme contemporain fasciné par lui-même : volonté d'intensification de soi au moyen d'une expérimentation aux limites de l'auto-annihilation, culture de la conservation de soi, augmentation (cybernétique, body building, vieillissement). Cette tyrannie disqualifie l'homme par désincarnation psychotique : «absent dans sa singularité, tout en étant hyper présent en tant que vecteur de dynamisation de l'univers image, est-il encore indispensable ? » (Gauthier, 2002, p. 54-55). Le choix d'une ou plusieurs identités de communication virtuelle de réseaux, blogs ou chat devient vital «car les interlocuteurs n'ont aucune certitude quant à l'identité affichée des uns et des autres, et donc quant à leur réalité. Le fait d'écouter quelqu'un et de lui parler (ou plus précisément de le lire et de lui écrire) fonctionne sur la présupposition qu'à l'autre bout du canal il y a quelqu'un que vous avez choisi d'accepter pour ce qu'il prétend être et dont vous attendez qu'il accepte ce que vous prétendez que vous êtes " (Schmoll, 2000, p. 44). C'est la fin de l'identité universellement objectivable de l'individu qui lui donne compétence pour interagir avec son milieu de vie, sa capacité citoyenne. Sans intervenir dans le débat public, devenu affaire de communication, il se contente de l'illusion hyper réelle de l'interaction. Revenu à une société de l'immanence, la transcendance divine, sociale ou politique tient lieu de destin à vocation consumériste.

La distinction entre ces deux niveaux d'analyse reste provisoire. Ce projet de société conservatrice s'efforce de disqualifier systématiquement les bases du projet moderne (autonomie et responsabilité de l'individu) et répond à une logique antidémocratique : les fortes implications politique et sociale de ces constructions hyper réelles véhiculent des propagandes sous-jacentes frontalement idéologiques. Le monde entier a pu s'y familiariser avec les images des bombardements de Bagdad lors des deux guerres irakiennes (Waks, 1994); déjà, Rambo 3 
(McDonald, 1988) choisissait son ennemi. Aujourd'hui, la propagande des milieux d'extrême droite américains utilise le vecteur de l'hyper-violence des jeux vidéo (Pilet, 2003) pour diffuser les thèses d'Huntington (identifier l'ennemi, construire la peur) et fonder ainsi la notion de civilisation (Crépon, 2002); dans la série américaine mondialement diffusée 24 heures, les méchants terroristes s'appellent bien sûr Marwan, Fayed, Assad ou, sinon, sont trafiquants de drogue mexicains, chinois ou ukrainiens...

Violente par son rythme, cette série l'est aussi par le viol de l'intimité que se permet le héros : véritable personnage de réalité augmentée, il est connecté en permanence à un réseau de recherche installé dans un QG hyper technologique qui lui fournit une information très précise (depuis le pistage de véhicules au moyen de la pulsation de téléphones portables jusqu'à la lecture par satellite de plaques d'immatriculation ou le pillage de fichiers): on dépasse les gadgets de James Bond, traités ironiquement, pour intégrer avec sérieux l'idée que ces images sont meilleures que ce que voit et vit le héros sur le terrain puisque ainsi rien ne lui échappe.

\section{Une violence soft consubstantielle à l'hyper réel}

La reprise du terme d'hyper réel se justifie parce que les espaces virtuels ne se limitent pas à l'Internet ou aux réseaux numériques. La nature métaphorique de ce processus d'hyper réalisation du monde rend cette violence implacable parce que soft $t^{10}$. Banal développement technique, le virtuel n'est pas dangereux en lui-même; c'est le processus d'hyper réalisation qu'il génère qui le transforme en «solution finale » (Doel, Clarke, 1999, p. 272) séduisante et perverse. «La machine transgresse la frontière entre l'animé et l'inanimé, viole les frontières du temps, se joue de la dimension symbolique de l'espace, elle s'auto-suffit dans un monde artificiellement recomposé. Elle arrache l'homme de sa condition non seulement spatio-temporelle mais encore sociale ou politique » (Gauthier, 2002 , p. 30). Les propos imprécatoires de Baudrillard ou pessimistes de Jameson (2007a) doivent être pris au sérieux. La dictature contemporaine du simulacre induit une perte de repères spatiaux et temporels, supprime toute mesure capable d'étalonner les sens, produit des espaces utopiques clos mal reliés entre eux et engendre ainsi une délocalisation des individus : «l'hyperespace postmoderne a

10 La mise en scène permanente de l'expérience des lieux produit des systèmes d'images dotés de leur propre autonomie : dans Alice, film de Marco Martins (2004), un père cherche sa petite fille disparue dans la ville; il la traque par l'image, celles de l'enfant (flyers et affiches) mais aussi de Lisbonne, grise et anonyme. Il plante des caméras dans les lieux fréquentés (carrefours, gares du métro, etc.). Elles lui donnent des ambiances glauques en noir et blanc. Le réalisateur explique que, faute de moyens pour payer des figurants, beaucoup de scènes furent tournées in vivo dans la rue à l'insu des passants. Lorsque l'acteur déambule dans la foule avec une affiche d'Alice, l'équipe s'interroge sur l'éventualité qu'un passant s'arrête et questionne l'acteur ; on décide d'improviser si le cas se présente. En quatre jours de tournage, cela n'est jamais arrivé. Derrière les icônes hyper-réelles du tram ou du fado dans la "Ville blanche », Lisbonne est une ville indifférente comme les autres (Crozat, 2007). 
finalement triomphé en dépassant la capacité du corps humain individuel à se localiser, à organiser son environnement immédiat » (Jameson, 2007a, p. 44).

Les trois niveaux de violence présentés en introduction sont reliés mais la pire violence est celle qui s'attaque au symbolique, en particulier celui des lieux. Les themscapes (Rodaway, 1994), scene (Bennett, Peterson, 2004) et autres hyper réalisations du local, par exemple touristique (Medina Lasansky, McLaren, 2004), minent l'enracinement, que Williams (1996) voyait comme antidote à l'uniformisation globalisante de Jameson (Bernard, 2003, p. 186). Contrairement à ce qu'affirmait aussi Santos (1997), la remédiation d'un local capable d'introduire le temps long pour interpréter l'instantanéité globale devient difficile :

L'enjeu d'aujourd'hui n'est donc pas une perte de mémoire [...] mais une perte d'individuation induisant des déplacements entre les instances d'individuation, comme il y en eut tout au long de l'histoire de l'humanité, avec ceci cependant que. Le risque d'entropie signifierait que non seulement l'instance d'individuation se déplace et se réorganise, mais qu'elle s'affaisse, c'est-à-dire que l'individuation comme néguentropie s'affaiblit du fait du conflit entre le psychique, le collectif et

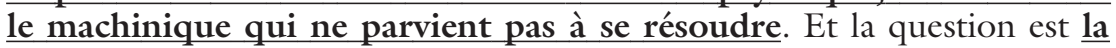
réorganisation de l'appropriation par l'ajustement de celle-ci à la nouvelle instrumentalité informationnelle des savoirs. Autrement dit. Le risque de la croissance de l'ignorance, plutôt que du savoir, serait celui d'un processus de désindividuation entropique par défaut d'appropriation correcte de la structure hypomnésique informationnelle (Stigler, 2005, p. 9 ; surligné par l'auteur).

Cette violence passe par trois étapes : la médiatisation instaure d'abord une confusion généralisée à finalité mercantiliste; celle-ci induit à son tour, un sentiment de délocalisation, perte de repères spatiaux et culturels (désorientation de Jameson ou Williams), source d'une souffrance diffuse qui est ensuite exploitée pour imposer une société de contrôle fondamentalement réactionnaire. Entre souffrance née de l'effritement de la dimension symbolique du monde et confusion identitaire, nous sommes «perdus dans l'espace»(Olalquiaga, 1992). Cette souffrance pousse les foules de consommateurs/citoyens dans l'hyper réalisation par la recherche d'ancrages territoriaux illusoires car factices : on n'a jamais créé autant de fêtes de la ruralité dans les périphéries lointaines des métropoles; elles n'ont jamais attiré autant de monde...

Mais certains tempèrent ce pessimisme : transitoire (Bell, 2001), la généralisation du virtuel ferait l'objet de lectures binaires Doel et Clarke (1999). La simulation produit-elle d'innocentes copies qui se rapprochent toujours plus du réel ou une matérialisation au rabais du refus de l'interaction sociale directe ? Le lien avec la représentation n'est pas toujours coupé ; l'extension vers de nouvelles expériences du monde offre des compétences accrues aux individus (Quéaud, 1993 ; Lévy, 1998 ; Hillis, 1999), re-médiatise et permet une meilleure compréhension du monde. "Au-delà de la rhétorique dominante de la mimesis, il est possible d'argumenter que la simulation établit la permanence de la nature 
représentationnelle d'une géographie du virtuel; on peut aller jusqu'à dire que, dépassant certaines craintes à leur sujet, les communications médiatisées par ordinateur n'instituent pas un ordre post-symbolique » (Crang et alii, 1999, p. 6). De plus, l'adaptation des sociétés à une réalité technique nouvelle, le développement de sociabilités spécifiques font évoluer les modalités de conflit social (Soja, 2001, p. 54) ou politique (Froehling, 1999).

Cependant, ces développements progressistes masquent mal des évolutions problématiques. En effet, l'enjeu de ces représentations des espaces, virtuels ou non, reste le pouvoir sur l'espace et les sociétés qui l'habitent : le propos central de cet article incite à ne pas sous-estimer la dimension sociale et politique des processus en cours. Le développement des systèmes de surveillance et de contrôle sociaux prolonge des mécanismes dont Foucault (1975) pointe l'apparition dès le $\mathrm{XVII}^{\mathrm{e}}$ siècle. Face donc à une simple évolution des sociétés, l'illusion de rupture proviendrait du basculement conservateur de la pensée française durant deux décennies (Cusset, 2003, p. 22, 323) et d'un changement de perspective avec la redécouverte contemporaine d'auteurs temporairement marginalisés (Foucault, Deleuze, Baudrillard).

Mais, des réalités virtuelles aux virtualités réelles, l'hyper réel n'est plus une copie ou une représentation; c'est une alternative opérant en parallèle avec le réel (Castells, 1998). Or, les développements qui précèdent insistent sur l'illusion d'interaction co-substantielle au virtuel. Perfection aristotélicienne du réel (Doel, Clarke, 1999, p. 268), l'hyper réel supprime le débat. La double volonté de transparence totale et de perfection, l'incapacité à la totalisation (Laurel, 1991 ; Doel, Clarke, 1999, p. 273) menacent la permanence de la topolitique de Derrida (1996) : l'organisation de l'espace et du temps du deuil, la relation à l'Autre, etc., ne sont plus pensées; c'est l'essence même du politique qui est en jeu. Nos sens sont manipulés pour générer des expériences géographiques réalistes. Combiné au recours à un éternel présent, cela restreint la possibilité de référer à la culture. La violence majeure est bien là.

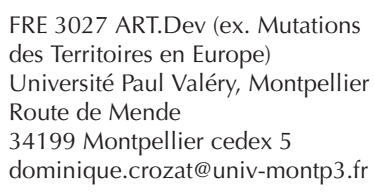

\section{Bibliographie}

Aubenas F., Benasayag M. (1999), La fabrication de I'information. Les journalistes et l'idéologie de la communication, Paris, La Découverte, 110 p.

Baudrillard J. (1981), Simulacres et simulation, réed. 1995, Paris, Galilée, coll. Débats, 233 p.

Baudrillard J. (1988), Amérique, Paris, Le Seuil, coll. Essai, 122 p.

Baudrillard J. (1995), Le crime parfait, Paris, Galilée, 207 p. 
Beck U. (2001 [1986]), La société du risque. Sur la voie d'une autre modernité, Paris, Aubier, 521 p. Bell D. (2001), An Introduction to Cyberculture, Londres, Routledge, 246 p.

Bennett A., Peterson R.A. (2004), Music scenes : local, translocal, and virtual, Nashville, Vanderbilt University Press, $264 \mathrm{p}$.

Bernal B. (2005), « El juego del miedo y la libertad. Un riesgo para la ciudad histórica », in O. Gutiérrez (coord.), La ciudad y el miedo, VII coloquio de Geografía Urbana, Barcelona, sept. 2004, Girona, Publicacions da Universitat de Girona, p. 153-168.

Bernard C. (2003), "Le canon moderniste à l'épreuve des cultural studies : à propos de The Politics of Modernism de Raymond Williams ", in A. Kaenel, C. Lejeune et M.-J. Rossignol (dir.), Cultural studies. Études culturelles, Nancy, Presses Universitaires de Nancy, p. 179-191.

Bobbé S. (2000), "Les nouvelles cultures du sauvage ou la quête de l'objet manquant. État de la question », Ruralia, n 7, p. 159-173.

Breton P. (1992), L'utopie de la communication. Le mythe du village planétaire, Paris, La Découverte, 172 p. (éd. 1997).

Brunel S. (2006), La planète disneylandisée. Chroniques d'un tour du monde, Paris, Sciences Humaines, $276 \mathrm{p}$.

Canteau L. (2003), Les écomusées en France, entre développement local, identité culturelle et lifting patrimonial, TER de maîtrise, Université Bordeaux 3, $114 \mathrm{p}$.

Castells M. (1998), La société en réseaux, Paris, Fayard, 613 p.

Charles S., Lipovetsky G. (2004), Les temps hypermodernes, Paris, Grasset, 186 p.

Châtelet G. (1998), Vivre et penser comme des porcs. De l'incitation à l'envie et à l'ennui dans les démocraties-marchés, Paris, Éditions Exils, éd. de poche (1999) Paris, Gallimard, coll. Folio actuel, $194 \mathrm{p}$.

Chevalier J., Carballo C. (2004), « Fermetures résidentielles et quête de l'entre-soi, entre Nord et Sud des Amériques ", L'Espace géographique, Dossier : Fragmentation urbaine, t. 33, 2004/4, p. 325-335.

Corbin A. et alii (1995), L'Avènement des loisirs (1850-1960), Paris, Aubier, 472 p.

Couchot E. (1998), La technologie dans l'art. De la photographie à la réalité virtuelle, Nîmes, Jacqueline Chambon, $269 \mathrm{p}$

Crang M., Crang P., May J. (1999) (éd.), Virtual Geographies : Bodies, Space and Relations, New York/Londres, Routledge, $322 \mathrm{p}$.

Crépon M. (2002), L'imposture du choc des civilisations, Nantes, Pleins feux, 83 p.

Crozat D. (2004), « Les contextes socio-territoriaux de la vie culturelle et de ses événements », Bulletin de l'Association de géographes français - Géographies, n² 2-2004, p. 242-255.

Crozat D. (2007a), « Thirdspace, espaces potentiels et hyper réel : nouvelles modalités de la fuite dans I'imaginaire ", in L. Viala et S. Villepontoux (dir.), Imaginaire, territoires, sociétés. Contributions à un déploiement transdisciplinaire de la géographie sociale, Montpellier, Publications de I'Université Paul Valéry Montpellier 3, p. 97-112

Crozat D. (2007b), «Ambiances et climats de Lisbonne: Hyper réalité, solitude et diversités d'une métropole moderne à travers la mutation de ses représentations artistiques », Sud-Ouest Européen, $\mathrm{n}^{\circ} 24-2007$, p. 39-50.

Crozat D., Raibaud Y. (2009), "La construction de l'image ethnique par la fête à Bordeaux ", in S. Fournier et alii, La fête au présent, Paris, L'Harmattan, p. 163-174.

Cusset F. (2003), "French Theory». Foucault, Derrida, Deleuze \& $C^{i e}$ et les mutations de la vie intellectuelle aux États-Unis, Paris, La Découverte, 367 p.

Davis M. (1997), City of Quartz. Los Angeles, capitale du futur, Paris, La Découverte, [1990], 393 p. 
Davis M. (2006), Au-delà de Blade Runner. Los Angeles et l'imagination du désastre, Paris, Allia, [1997], 154 p.

De Barros M. (2003), "Le virtuel incarné », in C. Buci-Glucksmann (dir.), L'art à l'époque du virtuel, Saint-Denis/Paris, Département Arts Plastiques, Université Paris 8/Éditions L'Harmattan, p. 171-186.

Derrida J. (1996), Échographies - de la télévision, Paris, Galilée.

Doel M., Clarke D.B. (1999), "Virtual worlds : simulation, suppletion, s(ed)uction and simulacra ", in M. Crang, P. Crang et J. May (éd.), Virtual Geographies : Bodies, Space and Relations, New York/Londres, Routledge, p. 261-283

Eco U. (1985), La Guerre du faux, Paris, Grasset, Le Livre de Poche-biblio, coll. Essais, 382 p.

Foucauld M. (1975), Surveiller et punir, Paris, Gallimard, 318 p.

Fournand A. (2007), "Quand l'image construit une spatialité corporelle, l'exemple de l'échographie fœtale », in L. Viala et S. Villepontoux (dir.), Imaginaire, territoires, sociétés. Contributions à un déploiement transdisciplinaire de la géographie sociale, Montpellier, Publications de I'Université Paul Valéry Montpellier 3, p. 39-50.

Fournier L., Crozat D., Bernié-Boissard C., Chastagnez C. (dir.), La fête au présent. Mutations des fêtes dans le temps des loisirs, Paris, L'Harmattan, coll. Travaux du CUFRN, 415 p.

Froehling O. (1999), «Internauts and guerrilleros. The Zapatista rebellion in Chiapas, Mexico and its extension into cyberspace», in M. Crang, P. Crang et J. May (éd.) Virtual Geographies : Bodies, Space and Relations, New York/Londres, Routledge, p. 164-177.

Gauthier A. (2002), Le virtuel au quotidien, Belfort, Circé, 207 p.

Giddens A. (1994), Les conséquences de la modernité, Paris, L'Harmattan, 192 p.

Hillis K. (1999), Digital sensations : Space, identity, and embodiment in virtual reality, Minneapolis, University of Minnesota Press, $271 \mathrm{p}$.

Hoyaux A.-F. (2004), "Pouvait-on habiter un camp de concentration sous le nazisme? », TIGR (Travaux de I'Institut de Géographie de Reims), nº 115-118, p. 123-136

Imken O. (1999), "The Global Matrix », in M. Crang, P. Crang et J. May (éd.), Virtual Geographies : Bodies, Space and Relations, New York/Londres, Routledge, p. 92-106.

Jameson F. (2007a [1991]), Le postmodernisme ou la logique culturelle du capitalisme tardif, Paris, École Nationale Supérieure des Beaux-Arts, 607 p.

Jameson F. (2007b), La totalité comme complot. Conspiration et paranoïa dans l'imaginaire contemporain, Paris, Les Prairies Ordinaires, 141 p.

Kitchin R. (1998), Cyberspace. The World in the wires, Chichester (UK), J. Wiley \& Sons, 214 p.

Klauser F. (2004), « La vidéosurveillance comme mécanisme de production disciplinaire de l'espace public. Une analyse empirique et théorique : I'exemple de la ville de Genève », Bulletin de I'Association de géographes français, décembre, vol. 81, n 4, p. 631-646.

Laurel B. (1991), Computers as theatre, Reading, Addison Wesley, 227 p.

Le Monde Diplomatique (2003), "Obsessions sécuritaires ", Manière de voir, nº 71.

Lévy P. (1998) Qu'est-ce que le virtuel ?, Paris, La Découverte, 153 p.

Lima (de) Gonçalves I. (2005), "La ciudad compleja : entre el miedo dirigido y las re-existencias », in O. Gutiérrez (coord.), La ciuada y el miedo, VII coloquio de Geografía Urbana, Barcelona, sept. 2004, Girona (Esp.), de l'Universitat de Girona : Server de Publicacions, p. 83-86.

López Levi L. (1999), Centros comerciales : espacios que navegan entre la realidad y la ficción, México, Editorial Nuestro Tiempo, 241 p.

Macé E. (2006), Les imaginaires médiatiques. Une sociologie postcritique des médias, Paris, Éditions d'Amsterdam, $168 \mathrm{p}$. 
Maigret E. (2005), «Après le choc des cultural studies», in E. Maigret, et E. Macé, Penser les médiacultures. Nouvelles pratiques et nouvelles approches de la représentation du monde, Paris, Armand Colin/INA, p. 17-40.

McGrath J. (2004), Loving Big Brother. Performance, privacy and surveillance space, Londres, Routledge, $246 \mathrm{p}$.

McLellan H. (1996), "Virtual realities », in D.H. Jonassen (éd.), Handbook of Research for Educational Communications and Technology. A Project of the Association for Educational Communications and Technology (AECT), New York, Simon \& Schuster Macmillan, p. 461-497.

Medina Lasansky D., McLaren B. (éd.) (2004), Architecture and Tourism. Perception, Performance and Place, Oxford, Berg, $320 \mathrm{p}$.

Metton A. (1997), "Espoirs et amertumes des commerces des centres-villes ", Les Annales de la Recherche Urbaine, $\mathrm{n}^{\circ}$ 1998-78, p. 87-93.

Micoud A. (2001), « La campagne comme espace public ? ", Géocarrefour, n 76, p. 69-73.

Miller D. (1997), Capitalism : an ethnographic approach, Oxford, Berg, 384 p.

Minca C. (2005), "The return of the Camp », Progress in Human Geography, 29, 4, p. 405-412.

Missonnier S., Lisandre H. (dir.) (2003), Le virtuel : la présence de l'absent, Paris, EDK Éditions, 264 p.

Mitchell D. (2001), « Postmodern Geographical Praxis? The Postmodern Impulse and the War against Homeless in the "Post-justice" City ", in C. Minca (éd.), Postmodern Geography. Theory and Praxis, Londres, Balckwell Publishing, p. 57-92.

Olalquiaga C. (1992), Megalopolis : contemporary cultural sensibilities, Minneapolis (USA), University of Minnessota Press, $113 \mathrm{p}$.

Percival J., Hanson J. (2006), « Big Brother or brave new world ? Telecare and its implications for older people's independance and social inclusion », Critical Social Policy, November, 1, 26(4), p. 888-909.

Perrin M. (2007), « Gouvernements résidentiels privés et municipalités détournées : I'habiter comme territoire affirmé, l'habiter comme sphère d'action collective ", in Ecole thématique internationale co-org. PACTE Grenoble, LATTS Paris, EPFL Lausanne, Les recompositions territoriales et les transformations de l'action publique. Trois énigmes sur la construction des intérêts territorialisés ; Thème 1 : Le territoire comme ressource pour l'action collective? ?ttp://www.pacte.cnrs.fr/IMG/ html_Controverses_Perrin.html.

Perrot M.-D. (1997), « Du vrai-faux au virtuel : mondialisation culturelle et néo-utopie », Nouveaux cahiers de l'Institut Universitaire du Développement, $\mathrm{n}^{\circ} 6$ : La mondialisation des anti-sociétés. Espaces rêvés et lieux communs, p. 41-63.

Pilet S. (2003), « Le jeu vidéo comme arme de propagande », Le Monde diplomatique, septembre 2003, p. 27.

Price P. (2000), "Inscribing the border », Social and Cultural Geography, vol. 1, n 1-2000, p. 101116.

Quéaud P. (1993), Le virtuel : vertus et vertiges, Seyssel, Champs Vallon, 215 p.

Quignard P. (1995), Rhétorique spéculative, Paris, Calmann-Lévy, éd. de poche, 1997, Gallimard/Folio, $198 \mathrm{p}$.

Quinz E. (2003), "Scènes virtuelles du corps », in C. Buci-Glucksmann (dir.), L'art à l'époque du virtuel, Saint-Denis/Paris, Département Arts Plastiques, Université Paris 8/Éditions L'Harmattan, p. 187-196.

Razac O. (1999), Histoire politique du barbelé, Paris, La Fabrique, 111 p.

Relph E. (1981), Rational Landscape and Humanistic Geography, Londres, Croom Helm, 279 p.

Reynaud L. (2006), "Demande sociale de risque zéro », in S. Fleuret, Espaces, qualité de vie et bien-être, Rennes, PUR, p. 29-35. 
Rifkin J. (2000), L'Âge de l'accès. La révolution de la nouvelle économie, Paris, La Découverte, 396 p. Rodaway P. (1994), Sensuous geographies, London, Routledge, 198 p.

Rodaway P. (1995), Exploring the subject in hyper-reality in Pile, S. ; Thrift, N. (éd.) Mapping the subject : geographies of cultural transformation, Londres, Routledge, p. 241-266.

Rodriguez I. (2005), " "Privatopia" versus Ciudad Pública ». La materialización del miedo en el espacio urbano, in O. Gutiérrez (coord.), La ciudad y el miedo, VII coloquio de Geografía Urbana, Barcelona, sept. 2004, Girona, Publicacions da Universitat de Girona, p. 127-152.

Rossi G. (2001), L'ingérence écologique. Environnement et développement rural du Nord au Sud, Paris, CNRS, $254 \mathrm{p}$.

Sack R.D. (1997), Homo geographicus, Baltimore, The John Hopkins University Press, 1997, p. 276, note 22.

Santos M. (1997), La nature de l'espace. Technique et temps, raison et émotion, Paris, L'Harmattan, $275 \mathrm{p}$.

Saunier P.-Y. (2004), "Tel Mickey Mouse jouant au tennis... », Note de recherche : Charles S. Ascher et l'internationalisation de la public administration, Actes de la recherche en sciences sociales, vol. Sociologie de la mondialisation, $\mathrm{n}^{\circ} 151-152,2004 / 1-2$, p. 49-56.

Schmoll P. (2000), «Les mondes virtuels, entre imagerie et imaginaire », Sociétés, 2000-4, n 70 , p. 33-46.

Schwarz M.J. (1994), Todos somos Superbarrio, Mexico, Grupo Editoral Planeta, 180p.

Sennett R. (1990), La ville à vue d'œil, Paris, Plon, 314 p.

Shields R. (2003), The Virtual, Londres, Routledge, 246 p.

Sloterdijk P. (1996), Essai d'intoxication volontaire, Paris, Calmann-Lévy, 185 p.

Soja E.W. (2000), Postmetropolis, Oxford, Blackwell, 440 p.

Soja E.W. (2001), "Exploring the Postmetropolis », in C. Minca (éd.), Postmodern Geography. Theory and Praxis, Oxford, Blackwell, p. 37-56.

Spring K. (2004), "Behind the Rave : Structure and Agency in a Rave Scene », in A. Bennett et R.A. Peterson (éd.), Music Scenes. Local, Translocal and Virtual, Nashville, Vanderbilt University Press, p. 48-63.

Stiegler B. (2004), De la misère symbolique. 1. L'époque industrielle, Paris, Galilée, 195 p.

Stiegler B. (2005), Ars Industrialis, séance de fondation (24 septembre 2005), Paris, Théâtre de la Colline. http://www.arsindustrialis.org.

Virilio P. (1984), L'espace critique, Paris, Christian Bourgois, 1984, 189 p.

Wark Mc K. (1994), Virtual geography. Living with Global Media Events, Bloomington/Indianapolis, Indiana University Press, $253 \mathrm{p}$.

Williams R. (1996), The Politics of Modernism, éd. Tony Pinkney, London, Verso. 